\title{
Managing System of Systems Requirements with a Requirements Screening Group
}

\section{INCOSE 2012}

\author{
Ronald R. Barden \\ David D. Evans
}

\section{July 2012}

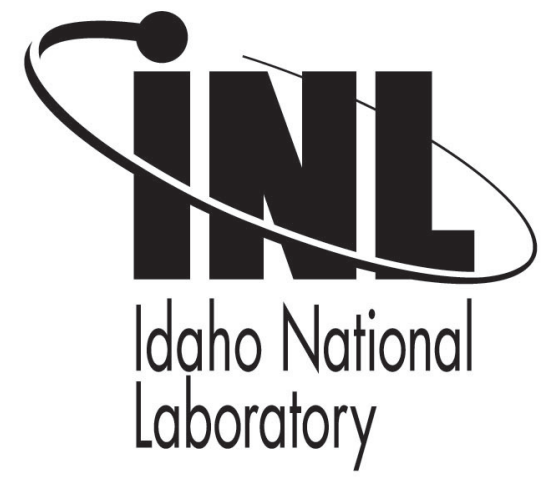

This is a preprint of a paper intended for publication in a journal or proceedings. Since changes may be made before publication, this preprint should not be cited or reproduced without permission of the author. This document was prepared as an account of work sponsored by an agency of the United States Government. Neither the United States Government nor any agency thereof, or any of their employees, makes any warranty, expressed or implied, or assumes any legal liability or responsibility for any third party's use, or the results of such use, of any information, apparatus, product or process disclosed in this report, or represents that its use by such third party would not infringe privately owned rights. The views expressed in this paper are not necessarily those of the United States Government or the sponsoring agency. 


\title{
Managing System of Systems Requirements with a Requirements Screening Group
}

\author{
Ronald R. Barden \\ Idaho National Laboratory \\ P.O. Box 1625, MS 3634 \\ Idaho Falls, Idaho 83415-3634 \\ (208) 526-8866 \\ ronald.barden@inl.gov
}

\author{
David D. Evans \\ Science Applications International Corp. \\ 1710 SAIC Cr., MS T1-9-9 \\ McLean, VA 22102 \\ (703) 676-5091 \\ david.d.evans@saic.com
}

Copyright @ 2012 by Battelle Energy Alliance, LLC. Published and used by INCOSE with permission.

\begin{abstract}
Figuring out an effective and efficient way to manage not only your Requirement's Baseline, but also the development of individual requirements during a System of Systems Program's Conceptual and Developmental Life Cycle Stages can be both daunting and difficult. This is especially so when you are dealing with a complex and large System of Systems (SoS) Program with many thousands of SoS Level Requirements as well as an even larger number of System, Subsystem and Configuration Item requirements that need to be managed. This task is made even more difficult when you add in integration of the efforts of multiple requirements development teams (e.g., Integrated Product Teams (IPTs)) and numerous System/Subsystem Design Teams.

One solution for tackling this difficult activity on a recent large System of Systems Program was to establish an empowered Requirements Screening Group (RSG). This group is a Team made up of co-chairs from the Contractor, Customer, and User Stakeholders that are empowered by and accountable to the Program Manager for Requirements Development on the Program. The RSG co-chairs, with the support of the System of System Requirements Team monitor, make decisions on, and provide guidance on all Requirements Development activities during the Conceptual and Development Life Cycle Stages of the Program. In addition, the RSG establishes and maintains the Requirements Baseline, monitors and enforces requirements traceability across the entire Program, and works with other elements of the Program to ensure integration and coordination.
\end{abstract}

\section{Introduction}

The problem in very large, complex programs is that often, the Operational Requirements Document (ORD) or Capability Development Document (CDD) are written in user terminology. That terminology, while meaningful to the user and customer (the acquisition organization), is often ambiguous and not well defined or perhaps not well understood by those outside the user community. Terms such as "quickly", "accurately", "safely", "user friendly" abound. Translating those into performance and behavioral requirements that can be used to build the resulting SoS and the individual component systems, and to measure the achievement of the requirement, is difficult and imprecise if there are many teams working mostly independently to develop performance and behavioral requirements and each team is applying 
its own interpretation of what the user wants. For very large, complex programs like the one cited as an example in this paper, a central entity is needed to provide consistent interpretation and guidance to all teams. One solution is an empowered Requirements Screening Group.

In a recent, exceedingly large and complex program for the US Army, more than 65,000 requirements at all levels from System of Systems to Configuration Item were generated. The Requirements Screening Group helped ensure consistency, accuracy, and integration. One of its most important contributions, was the interpretation of user intent of the operationally worded ORD (and later CDD) requirements. Another major contribution was to be the "referee" of requirements. The RSG ensured that no IPT could levy conflicting requirements on another. Finally, the RSG ensured that all requirements at the SoS and system level passed the "sniff test", or "giggle test" (e.g., "The [19 ton] cannon shall hover and stare in 20 knot winds."). This meant overseeing the requirements development process and ensuring that some intellectual content went into the development of each requirement, not just a rote assembly line production.

The solution was the creation of a Requirements Screening Group (RSG), which had oversight responsibility for all phases of requirements development and maintenance for the Program. Activities for which the RSG had responsibility and oversight included:

- Interpretation of user intent as guidance for requirements development

- Stakeholder requirements development and documentation

- SoS Performance/technical requirements development and documentation

- Requirements traceability between the stakeholder requirements document and SoS and individual system specifications

- Traceability between SoS requirements and individual system requirements

- Stakeholder requirements change requests

- Requirements review and approval.

- SoS performance/technical specification change requests

- Requirements prescreening for requirements' changes to be made by Leadership

- Recommendations of changes to the Program-level Requirements Document (e.g. Operational Requirements Document [ORD], Capability Development Document [CDD], A-level Specification [A-Spec])

- "Traffic cop" - ensuring requirements from one group did not contradict or impose an unreasonable burden on another

The concept for the RSG was that it would be composed of members from each major stakeholder group in the Program. In the case of the recent example discussed above, this group was comprised of members from the User group, the Customer Group (Acquisition) and the Lead Systems Integrator (Prime Contractor). As such, the RSG is a triumvirate made up of a Senior individual from each of these three stakeholders, empowered by their leadership, each with an equal vote on RSG issues. In addition, because of the sheer number of requirements and volume of management required, each co-chair had a team they could use to support the numerous and detailed RSG tasks.

This paper will provide a general summary of the approximately 6 year run of this Major Program's RSG. It will describe how the RSG was initially formed, what was necessary to get requirements development activities started, requirements maintenance precedence, RSG 
Products/Milestones/Reports and finally some conclusions about making use of an RSG for other programs' requirements development and maintenance activities. In terms of Group/Team dynamics development phases, first created by Psychologist Bruce Tuckman, RSG development and operation will be categorized by the following: Forming, Storming, Norming and Performing.

For reference purposes, Bruce Tuckman's four Stages of Team development phases are Forming, Storming, Norming, and Performing. During the Forming stage, the team energy and effort is typically spent defining the mission, setting objectives, defining responsibilities, and establishing the team process and schedule with deadlines. In the Storming stage, the team will begin to work together to achieve shared goals/results/responsibilites, to further refine goals/results/responsibilities, or to achieve new goal/responsibilities/results. Team development progress continues in the Norming stage, in which team members begin to accept their roles and responsibilities and to adjust their behavior toward each other as they develop habits that make teamwork seem more natural and fluid. Norming comes from the word normal and implies everyone working together with both their enthusiasm and collective skills increasing and aligning to match the work to be done. Finally, during the Performing stage, the primary focus is on performance, where purpose, roles, responsibilities and goals are clear; standards are high; and there is a commitment to not only meeting baseline standards but to continuous improvement. Also during Performing, communication is open and leadership is shared.

\section{Forming}

When the Army's Program Manager decided to use an RSG, one of the most important tenants of success was empowering the board with both significant autonomy and decision-making authority. Obviously, there were still high level program requirements decisions that Program leadership made, but Program leadership did not have time, nor did it want to be burdened with most day to day requirements decisions. An empowered RSG handled all of the requirement development and change activities for a large majority of the Program's requirements. Those requirements changes that affected performance, cost or schedule were referred to the Program Executive Steering Group by the RSG, along with an accompanying RSG recommendation. In this way Program leadership was free to deal with the multitude of other Program issues. Consequently, decision-makers chairing the RSG had to be knowledgeable about Requirements development methods and practices, requirements traceability, and Specification development, as well as knowledgeable enough about the Program to be able to make timely decisions and move the Program forward.

The first step in establishing the RSG was to select the RSG co-chairs. It is recommended that a single, empowered co-chair be assigned from each of the major stakeholder groups (e.g. User, Acquisition, Prime Contractor). In this case, the co-chairs were the Army's head of the Material Requirements Division (representing the User), the Prime Contractor's lead for System of Systems Requirements development, and the Customer's Senior System of Systems Engineer (representing the Acquisition organization). Each RSG co-chair brought with them a support team. It is difficult for a single individual to effectively handle all Requirements Development activity for a large complex Program. A key factor for success was that each RSG co-chair was empowered by their organization to make decisions related to requirements development for the program. 
The RSG co-chairs drafted a charter and presented it to the Program Review Board for approval. The RSG charter documented the purpose and authority of the RSG and the conditions under which it will operate. Included in the charter is a description of the authority and responsibilities of each co-chair, support team members, and anticipated RSG Activities. In addition, RSG Processes were developed to document how the RSG operated. An RSG Process should provide guidance, at a minimum, for the following activities: 1) Requirements development; 2) Requirements Management; 3) Requirements change process; 4) Specification development/maintenance; 5) Configuration Management (Requirements Baseline); 6) Traceability and; 7) conduct of RSG technical meetings (official and ad hoc).

While the User requirements document contains User need statements and strictly belongs to User, it is recommended that the RSG team support refinement of the User Need Document. With some knowledge and unique perspective from respective design teams, the Acquisition and Prime Contractor co-chairs should be able help balance the descriptions of the User need statements, possibly helping to avoid conflicts later on both the requirements development and the design processes. Experience has shown that it is better that the User need statement document not include "shall" statements, generally attributable to technical/performance requirements in the design development phase, to avoid confusion between these Need statements and technical/performance requirements. In addition, it is recommended that each User need statement include two levels of anticipated performance: Threshold and Objective. Threshold needs are the minimum capability that the User is expecting for a given area. Objective needs are generally described as goals, which allow for possible growth during the design phase of the Program. Also, keep in mind that the User Needs Document should be describing desired User capabilities from an Operational perspective. It should not be attempt to dictate the design.

\section{Storming}

Proper translation of User Need Capabilities into Behavioral and Performance Requirements is critically important and should have adequate resources and time allocated. A single Operational Need Capability statement can translate into many individual technical requirements as the capability is decomposed into its performance, behavioral, and functional components. The large complex System of Systems Program in this case had well over 10,000 System of Systems and System requirements derived from a little more than 550 User Need capability statements in the ORD. One issue encountered early in the program was that many of the individual System IPT requirements developers had minimal experience in developing requirements. They tended to copy each Operational Need statement, with cosmetic changes to make it appear like a Technical performance requirement. Had the RSG not intervened, it would have lead to poor requirements, a faulty design, and a high likelihood of failure of the Program. The RSG support team conducted many group and individual sessions to teach requirements development and ensure each IPT understood what constituted a good requirement. This same training was offered to User and Acquisition personnel early in the program and resulted in a better understanding of the requirements development process. The RSG co-chairs performed an oversight role of the efforts of more than 200 requirements developers from the System of Systems level to the individual Configuration Item level. This was successful because the RSG co-chairs were knowledgeable and experienced in the Requirements development process, which enabled them to provide consistent guidance to the various Requirements development teams. 
Once the System of Systems Specification requirements set was reasonably stable, decomposition of the requirements and allocation to the Lower level (System, subsystem, Configuration item, etc.) specifications began. The RSG and its support team continued to operate mostly in an oversight and clarification role through the Preliminary Design Review, and, with a diminishing role, to the Critical Design Review phase. Emphasis shifted more toward traceability and preventing requirements conflicts at the lower levels.

Equally important during this timeframe was RSG involvement in and awareness of the Baseline and Configuration Management Processes. With tens of thousands of requirements in various stages of development and numerous Program specifications concurrently in development, it was important for the RSG to understand what their current Requirements Baseline was and how that baseline was being Configuration Managed.

\section{Norming}

As the Program matured, the RSG shifted from mostly leading and educating to an oversight and advisory role, accomplished through both scheduled and ad hoc reviews. A major RSG Review was the User Capability Coverage Review. This review was an extensive review of traceability between the User Capability Needs document and the defined Behavioral and Performance requirements in the System of Systems Specification. As the program emphasis

shifted to the individual Systems, this same type of review was preformed for each set of system level requirements, identifying traceability from the System to the System of Systems requirements and to the User Capability Development Document. Since the Program was particularly large and complex, teams of the various Stakeholders members often conducted a targeted capability by capability assessment of the requirements against the stated desired capability. The results of these assessments were presented to the RSG, who had final authority to determine the degree of coverage (successful traceability). In cases where a program is more mature or has fewer requirements, the RSG could undertake this activity directly. If there were disagreements among the RSG co-chairs on a particular traceability call, a simple majority vote determined the final coverage status. Results, gaps, issues, and directions from this coverage review were documented by the RSG and reported to the Program leadership. Each User need capability was assessed as having full coverage, partial coverage, or no coverage when compared against the User capability requirements and the System of Systems requirements. Full coverage meant that a complete set of performance and behavioral requirements covered the User capability in question. Partial coverage indicates that performance requirements exist to cover only a portion of the User capability, and additional requirements will be needed to fully cover the capability in question. No coverage infers that there are no current requirements that help meet the desired User capability. As the Program matured, a continuing no coverage or a partial coverage assessment meant the Program needed to re-examine the basis for the User's stated capability and whether current or anticipated technology will enable achieving the stated need.

It should be noted that RSG Traceability exercises are not just limited to ensuring traceability between the User document and the Program Top Level Performance Specification. RSG must also keep track of requirements decomposition and definition between the Top Level Requirements it has been given responsibility for and lower level System level and below requirements. Integrated Product Teams (IPTs) often have responsibility for developing these lower level requirements. In order to maintain consistency and traceability between requirements sets, there should be close interaction with the RSG, who knows and understands the impacts on development of the lower level requirements to the highest level requirements 
and the User Requirements. IPTs should make use of the RSG Board and expertise to assess traceability of requirements and ensure there are no violations in requirements definition and decomposition activities. After all, the final design is based on the derived performance and behavioral requirements and must meet the needs and capabilities laid out by the User Needs.

The RSG Charter established a responsibility to report weekly to the Program leadership on the results of its meetings, reviews and activities. During Program design requirements development stage, it is vitally important that all requirements developers are aware of the current requirements baseline, any User requirements changes under consideration, and any implemented changes to the User Need Document or the System of Systems specification. Program leadership found that metrics provided by the RSG on traceability and coverage results were a good gauge of how well the Program is doing against the documented User need capabilities. All RSG requirements reviews and other meetings, per the RSG charter, were documented in meeting minutes so there was an historical record of decisions made and lessons learned. Experience showed that it was vitally important that all RSG activities were documented and searchable for historical purposes because, with a large and long term program, personnel turnover on all sides makes it difficult to keep track of all decisions, issues and activities over the length of the Program.

During the Norming phase, the RSG found itself having to propose changes to the User Capability Needs document, some of which could only be approved by the Chief of Staff of the Army. These changes, once approved, then rippled down through the various levels of requirements documents in the Program.

As the Program progresses and matures, there will be changes to the way the Program does business. The RSG has to be flexible and adaptable to these Program changes to meet the needs of the Program. In this case, the RSG periodically reviewed its own Process against changes in the overall Program process set. .

It is recommended that regular RSG meetings be scheduled on monthly or bi-weekly basis with ad hoc meetings scheduled as the need arises. These regular RSG meetings should have a preset agenda, have all co-chairs or their appointed representative present, be constructive, and have a documented set of meeting minutes published.

\section{Performing}

As the Program gained momentum, the RSG found itself spending a large majority of its time on Requirements Gaps and Issues or dealing with significant requirements changes to the Top Level Specification and User Need Document. This was largely due to the fact that the Program was satisfied with its requirements sets and preliminary design against most of the documented User need capabilities and could focus on those that presented the greatest challenge in terms of technology, cost, and schedule.

Regular and annual Traceability and Coverage reviews continued to be conducted by the RSG and metrics reported to Program leadership, but these were becoming fairly routine. Comprehensive reviews were scheduled in advance of major Program Reviews and results reported at the Program Reviews. The RSG found itself dealing more and more with a narrower range of Requirements issues and gaps as the Program matured. Working these Issues and Gaps required the RSG to dig deeper into design issues, often calling in specific Subject Matter Experts (SMEs) from the Contractor, User, and Acquisition communities to 
help find ways to mitigate the issue or gap. The RSG was additionally tasked with tracking status and progress on all issues and gaps.

As the Program approached PDR, the RSG found itself focusing as much on schedule as it did on requirements. The Program Master Schedule of major reviews and milestones dictated how often the RSG needed to baseline its requirements sets and specifications. The RSG schedule and Milestone acceptance criteria (during a formal program review) were integrated into the Program Master Schedule.

From the RSG's inception through PDR, the RSG found itself asked to participate as a preliminary screening group for the Program decision-makers. It became obvious, that certain requirements and the resulting preliminary design would have a huge programmatic impact. These types of items are usually documented as the Major Programmatic gaps/issues. While these Programmatic Issues were beyond the RSG Charter to resolve, Program leadership felt the RSG had the most comprehensive understanding of the causes and effects. Rather than attempting to wade through the entire history of the major issues and gaps, Program leadership asked the RSG to "prescreen" details (technical, cost, schedule) and provide an integrated, recommended position to Program leadership on the each Issue or Gap. Program leadership then took that recommendation under advisement and rendered a decision, along with Program direction. In all fairness, it should be noted that not all RSG recommendations were accepted, but those occasions were in the very small minority.

\section{Conclusion}

Having to manage a requirements' set, a requirements baseline, ensuring requirements traceability and establishing specifications for a complex System of Systems or even a System is a very difficult and daunting prospect. This is especially true if about the Program faces the prospect of thousands of Top level requirements, and many more lower level requirements. Establishing and empowering an accountable and responsible Requirements Screening Group is one way to manage this difficult activity for the Program. An integrated, organized, coordinated and empowered RSG provides the necessary requirements oversight and expertise for the Program. RSG activities will diminish after the Preliminary Design Review (PDR) and phase out almost entirely by the Critical Design Review (CDR). There will be some housekeeping and archival activities after that but for all intents and purposes, the RSG will stand down when production or implementation phases in. One strong caveat however. For this concept to work successfully, it requires buy-in from Program leadership and empowered, knowledgeable, and capable RSG co-chairs and support teams. It works best on large, diverse, complex programs, but the concept can be applied on many smaller, less complex programs.

The RSG for the large and complex System of Systems Program in this case had the following lessons learned:

- Make sure the RSG is, in fact, a whole team effort. The RSG, including the support teams and especially the various co-chairs, needs to be committed to development of high quality requirements sets for the Program, establishment of the requirements' baselines, and maintenance of requirements traceability and working of requirements issues and gaps.

- The RSG must be empowered by the Program leadership to make decisions on requirements. 
- The RSG should have detailed and documented processes for its various activities and meetings to ensure consistency in dealing with requirements issues as well as to document lessons learned for future RSG members and programs.

- While RSG co-chairs do not have to necessarily agree on all issues, they must respect the positions of each other and abide by the majority decision. Significant progress on requirements development will be severely hindered if this mutual respect is not present.

- Do not give in to pressure for changes to requirements too early in the design/development process. Experience has shown that early relief on stated requirements can limit preliminary design solutions, constrict the design trade space, and can produce unforeseen limits on complimentary requirements. Then too, the pace of technology development may render the problem moot well before it was necessary to make a hard decision in the design process. Requirements waivers, deviations and changes can postponed until later in the Program for many particularly difficult requirements yet not cause undue impact on cost or schedule.

- It was found important to conscientiously document and publish all RSG decisions and meeting minutes so as to have a good historical basis on which to base future RSG requirements decisions. This also captured the corporate knowledge of Program members who transitioned out of the Program.

- It is a good idea for the RSG to publish its charter and processes early, to have regularly scheduled meetings and reviews to address the large amount of requirements changes that occur on large complex Programs, and above all, to remain flexible to meet the critical needs of the Program.

\section{Acknowledgements}

This manuscript has been authored by Battelle Energy Alliance, LLC under Contract No. DE-AC07-05ID14517 with the U.S. Department of Energy. The United States Government retains and the publisher, by accepting the article for publication, acknowledges that the United States Government retains a nonexclusive, paid-up, irrevocable, world-wide, license to publish or reproduce the published form of this manuscript, or allow others to do so, for United States Government purposes.

\section{References}

Tuckman, Bruce W. (1965) "Developmental sequence in small groups," Psychological Bulletin, 63, 384-399. Reprinted in Group Facilitation: A Research and Applications Journal, Number 3, Spring 2001.

\section{Biography}

Ronald R. Barden currently works as a Systems Engineer in the Systems Engineering Department at the Idaho National Laboratory (INL) in Idaho Falls, Idaho. Ronald has a B.S. degree in Mechanical Engineering from the University of Colorado, Boulder and has worked at the INL for over 12 years. Ronald has over 25 years of experience in Systems Engineering activities for both large and complex System of Systems Programs and small individual Projects. This Systems Engineering experience includes extensive work on requirements development, functional decomposition, trade studies, integration, project scheduling and design progress assessment. In addition to various INL Projects, Ronald has supported programs/projects for the U.S. Army, the Department of Defense, the Department of Energy, 
the U.S. Air Force and several large U.S. Defense Contractors. Ronald is also an INCOSE Certified Systems Engineering Profession (CSEP) and possesses the US Department of Defense (DoD) Acquisition Extension in conjunction with the CSEP (CSEP-Acq) base certification.

David D. Evans is employed by Science Applications International Corporation (SAIC) as Chief Engineer for the Systems and Technology Solutions Business Unit. Mr. Evans has 40 years of System Engineering and Integration experience, nearly 15 years of that with SAIC. He was the System of Systems Requirements lead for the Future Combat System (FCS) program, System Engineer and Requirements lead for NASA's Vibro-acoustic Test Capability (VTC), and Program Manager and Chief Engineer for the prototype Patriot Tactical Ballistic Missile Defense system (later JTAGS). Mr. Evans has a B.S. in Chemistry from The George Washington University. 